PROCEEDINGS OF THE

AMERICAN MATHEMATICAL SOCIETY

Volume 133, Number 3, Pages 773-785

S 0002-9939(04)07532-X

Article electronically published on August 20, 2004

\title{
EXAMPLES CONCERNING HEREDITY PROBLEMS OF WCG BANACH SPACES
}

\author{
SPIROS A. ARGYROS AND SOPHOCLES MERCOURAKIS
}

(Communicated by N. Tomczak-Jaegermann)

\begin{abstract}
We present two examples of WCG spaces that are not hereditarily WCG. The first is a space with an unconditional basis, and the second is a space $X$ such that $X^{* *}$ is WCG and $X^{* *}$ does not contain $\ell^{1}$. The non-WCG subspace $Y$ of $X$ has the additional property that $Y^{* *}$ is not WCG and $X / Y$ is reflexive.
\end{abstract}

\section{INTRODUCTION}

Thirty years ago H. P. Rosenthal ( $[\mathbb{R}]$ ) showed that there exists a non-WCG subspace of $L^{1}(\mu)$, with $\mu$ an appropriate probability measure, settling in the negative the heredity problem for WCG spaces. The aim of the present paper is to provide some more examples related to some heredity questions stated in the literature. More precisely our examples concern the following.

$\mathbf{Q}_{\mathbf{1}}$ : Is every WCG space with an unconditional basis hereditary WCG?

This is a well-known problem recently stated in $[\mathrm{Z}$.

$\mathbf{Q}_{\mathbf{2}}$ : If $X^{* *}$ is WCG, does $X$ share the same property?

This problem was posed by W. Johnson and J. Lindenstrauss JL.

$\mathbf{Q}_{3}$ : Is every WCG space not containing $\ell^{1}$ hereditarily WCG?

K. John and V. Zizler have shown that WCG with a Fréchet smooth norm are hereditary WCG [JZ]. M. Fabián has extended this result [F1]. Thus $\mathbf{Q}_{\mathbf{3}}$ concerns the weaker assumption that $\ell^{1}$ does not embed into $X$. Let us mention that in [MS] it has been shown that under Martin's axiom every WCG space with $\operatorname{dim} X<2^{\omega}$ is also hereditarily WCG.

In the first section, answering $\mathbf{Q}_{\mathbf{1}}$, we present an example of a WCG space $X$ with an unconditional basis that has a non-WCG subspace $Y$. Additionally $Y$ is generated by a block basis of $X$ and thus it also has an unconditional basis. This example is unexpectedly easy and is built on the well-known Talagrand's compactum. However it shares some remarkable properties. Thus the unconditional basis of $X$ is decomposed into countably many pieces, each one generating a Hilbert space. On the other hand, the unconditional basis of the subspace $Y$ is extended to an $M$-basis of $X$ failing Johnson's property (i.e. there is no countable partition of it with each piece being a relatively weakly compact set) and which also is decomposed into countably many pieces, each one generating a non-WCG subspace of $X$.

Received by the editors July 16, 2003 and, in revised form, October 23, 2003.

2000 Mathematics Subject Classification. Primary 46B20, 46B26, 03 E05.

Key words and phrases. WCG Banach space, unconditional basis, tree.

(c)2004 American Mathematical Society 
The second section is combinatorial in nature. It contains one of the basic ingredients of the second example. In particular we show the following.

Theorem. There exists a sequence $\left(T_{n}\right)_{n}$ of trees satisfying the following:

(i) Each $T_{n}$ is of height $\omega$ and for $n_{1} \neq n_{2}$, if $S_{i}$ is a segment of $T_{n_{i}}, i=1,2$, then $\left|S_{1} \cap S_{2}\right| \leq 1$.

(ii) For every partition $\left(D_{n}\right)_{n}$ of $T=\bigcup_{n} T_{n}$ there exists $n_{0} \in \mathbb{N}$ such that $D_{n_{0}} \cap T_{n_{0}}$ contains an infinite segment of $T_{n_{0}}$.

We actually show that the remarkable Rezničenko sequence of trees satisfies the above-stated properties.

In the third section we present a Banach space $X$ with the following properties.

(i) Both $X$ and $X^{* *}$ are WCG and do not contain $\ell^{1}$.

(ii) There exists a subspace $Y$ of $X$ such that both $Y$ and $Y^{* *}$ are not WCG.

(iii) The quotient space $X / Y$ is reflexive.

This example answers in the negative $\mathbf{Q}_{\mathbf{3}}$ and is also related to $\mathbf{Q}_{\mathbf{2}}$, which still remains open. The space $X$ is of the form $\left(\sum \oplus X_{n}\right)_{2}$ where each $X_{n}$ is James's space $J\left(T_{n}\right)$ and $\left(T_{n}\right)_{n}$ is a Rezničenko sequence. The unconditional counterpart of this example provides another example of a space with an unconditional basis containing a non-WCG subspace $Y$ with the additional property that $X / Y$ is reflexive. We finally mention that in the first section we provide a necessary property, weaker than Johnson's property, satisfied by any $M$-basis of a WCG space.

\section{THE HEREDITY PROBLEM FOR SPACES WITH AN UNCONDITIONAL BASIS}

In the first part of this section we prove the aforementioned property of $M$-bases. This will be used for checking the non-WCG property in the case of spaces without unconditional basis. In the second part we present the first of our examples.

Proposition 2.1. Let $X$ be a WCG Banach space, and let $\left\{\left(x_{a}, f_{a}\right): a \in T\right\}$ be any $M$-basis of $X$. Then there exist a sequence $T_{m}, m \geq 1$, of subsets of $T$ with $T=\bigcup_{m=1}^{\infty} T_{m}$ and a partition $\left\{T_{\delta}: \delta \in \Delta\right\}$ of $T$ into countable sets such that, if $\left(a_{n}\right) \subseteq T$ and $\left(\lambda_{n}\right) \subseteq \mathbb{R}$ with the properties:

(a) $a_{n} \in T_{\delta_{n}}, n \geq 1$, for some sequence $\left(\delta_{n}\right)$ of distinct points of $\Delta$ and

(b) $\liminf _{n \rightarrow \infty}\left\|\sum_{k=1}^{n} \lambda_{k} f_{a_{k}}\right\|<+\infty$,

then we have, for every $\varepsilon>0$ and $m \in \mathbb{N}$ that the set

$$
\left\{n \in \mathbb{N}: a_{n} \in T_{m} \text { and }\left|\lambda_{n}\right| \geq \varepsilon\right\} \quad \text { is finite. }
$$

Proof. It is clear that the set $K=\left\{\frac{f_{a}}{\left\|f_{a}\right\|}: a \in T\right\}$ is $w^{*}$ discrete with $K \cup\{0\} w^{*}$ compact. Since $X$ is WCG there exists a weakly discrete total subset $\left\{y_{\gamma}: \gamma \in \Gamma\right\}$ of $X$ so that the set $\left\{y_{\gamma}: \gamma \in \Gamma\right\} \cup\{0\}$ is weakly compact (see $\mathrm{AL}$ ). We define a function $F: T \times \Gamma \rightarrow \mathbb{R}$, by the rule $F(\alpha, \gamma)=f_{a}\left(y_{\gamma}\right)$, for $\alpha \in T, \gamma \in T$ and easily conclude that for any pair $(\alpha, \gamma) \in T \times \Gamma$ both of the sets $\left\{\alpha^{\prime} \in T: F\left(\alpha^{\prime}, \gamma\right) \neq 0\right\}$ and $\left\{\gamma^{\prime} \in \Gamma: F\left(\alpha, \gamma^{\prime}\right) \neq 0\right\}$ are at most countable. By applying a result of the first-named author (see [A] or ACGJM Lemma 2.4 or Lemma 1.6.2 in [F]), we can find partitions $\left\{\Gamma_{\delta}: \delta \in \Delta\right\}$ and $\left\{T_{\delta}: \delta \in \Delta\right\}$ of $\Gamma, T$ into countable sets with the following property: For every $\delta_{1}, \delta_{2} \in \Delta, \delta_{1} \neq \delta_{2}$, and for every $\alpha \in T_{\delta_{1}}$ and $\gamma \in \Gamma_{\delta_{2}}$, we have $f_{a}\left(y_{\gamma}\right)=0$. 
Now set $T_{m}=\left\{\alpha \in T\right.$ : there exists $\delta \in \Delta$ with $\alpha \in T_{\delta}$ and $\gamma \in \Gamma_{\delta}:\left|f_{a}\left(y_{\gamma}\right)\right| \geq$ $\left.\frac{1}{m}\right\}$, for $m \geq 1$; then clearly $T=\bigcup_{m=1}^{\infty} T_{m}$. We shall show that the families $\left\{T_{m}: m \in \mathbb{N}\right\}$ and $\left\{T_{\delta}: \delta \in \Delta\right\}$ satisfy the desired properties. So let $\left(\alpha_{n}\right) \subseteq T$ and $\left(\lambda_{n}\right) \subseteq \mathbb{R}$ having properties (a) and (b) of the theorem. Assume that there exist $\varepsilon>0, m_{0} \in \mathbb{N}$ and a subsequence $\left(\alpha_{k_{n}}\right)$ of $\left(\alpha_{n}\right)$ so that $\left\{\alpha_{k_{n}}: n \geq 1\right\} \subseteq T_{m_{0}}$ and $\left|\lambda_{k_{n}}\right| \geq \varepsilon$, for all $n \geq 1$. Let $\left(\gamma_{n}\right)$ be a sequence of (necessarily distinct) points of $\Gamma$ such that $\left|f_{\alpha_{k_{n}}}\left(y_{\gamma_{n}}\right)\right| \geq \frac{1}{m_{0}}$ for all $n \geq 1$. Property (b) and the fact that any closed ball of $X^{*}$ is an Eberlein compact set in the $w^{*}$ topology yield that there exists a subsequence $\left(x_{\mu_{n}}^{*}\right)$ of the sequence $x_{n}^{*}=\sum_{k=1}^{n} \lambda_{k} f_{a_{k}}, n \geq 1$, with $x_{\mu_{n}}^{*} \stackrel{w *}{\rightarrow} x^{*} \in X^{*}$. Fix any $\nu \in \mathbb{N}$. Then we have (by also using property (a)) that, $\left|x *\left(y_{\gamma_{\nu}}\right)\right|=\lim _{n \rightarrow \infty}\left|\left(\sum_{k=1}^{\mu_{n}} \lambda_{k} f_{\alpha_{k}}\right)\left(y_{\gamma_{\nu}}\right)\right|=\left|\lambda_{k_{\nu}}\right| \cdot\left|f_{\alpha_{k_{\nu}}}\left(y_{\gamma_{\nu}}\right)\right| \geq \frac{\left|\lambda_{k_{\nu}}\right|}{m_{0}}$, for large $n$. Therefore, $\left|x^{*}\left(y_{\gamma_{\nu}}\right)\right| \geq \frac{\left|\lambda_{\nu}\right|}{m_{0}} \geq \frac{\varepsilon}{m_{0}}$, for all $\nu \in \mathbb{N}$, which contradicts the fact that $y_{\gamma_{\nu}} \stackrel{w}{\rightarrow} 0$. The proof of the proposition is complete.

Theorem 2.2. Let $X$ be a WCG Banach space, and let $\left\{\left(x_{\alpha} \cdot f_{\alpha}\right): \alpha \in T\right\}$ be any $M$-basis of $X$. Then there exists a sequence $D_{m}, m \geq 1$, of subsets of $T$ with $T=\bigcup_{m=1}^{\infty} D_{m}$ such that, if $m \in \mathbb{N},\left(\alpha_{n}\right)$ is any sequence of distinct points of $D_{m}$ and $\left(\lambda_{n}\right)$ is any sequence in $\mathbb{R}$ with $\liminf _{n \rightarrow \infty}\left\|\sum_{k=1}^{n} \lambda_{k} f_{a_{k}}\right\|<+\infty$, then $\lambda_{n} \rightarrow 0$.

Proof. Let $\left\{T_{m}: m \in \mathbb{N}\right\}$ and $\left\{T_{\delta}: \delta \in \Delta\right\}$ be families as in the statement of the above theorem. Let, for $\delta \in \Delta, T_{\delta}=\left\{\alpha_{1}^{\delta}, \ldots, \alpha_{n}^{\delta}, \ldots\right\}$ be a one-to-one enumeration of $T_{\delta}$ and for $k \in \mathbb{N}, E_{k}=\left\{\alpha \in T: \alpha=\alpha_{k}^{\delta}\right.$, for some $\left.\delta \in \Delta\right\}$. We also set $T_{n, k}=T_{n} \cap E_{k}$, for $n, k \in \mathbb{N}$, hence $T=\bigcup_{n \geq 1, k \geq 1} T_{n, k}$. It is clear that the countable family $\left\{T_{n, k}: n, k \in \mathbb{N}\right\}$, which we enumerate as $\left\{D_{m}: m \in \mathbb{N}\right\}$, satisfies the conclusion of the theorem.

An easy consequence of the above theorem is the well-known characterization of WCG Banach spaces having an unconditional basis due to Johnson (see $[\underline{\mathrm{R}}$ and [F]).

Theorem 2.3 (Johnson). Let $X$ be a WCG Banach space with an unconditional basis $\left\{x_{\gamma}: \gamma \in \Gamma\right\}$. Then the following are equivalent:

(i) $X$ is $W C G$.

(ii) There exists a sequence $\Gamma_{n}, n \geq 1$, of subsets of $\Gamma$ with $\Gamma=\bigcup_{n=1}^{\infty} \Gamma_{n}$ so that each set $\left\{x_{\gamma}: \gamma \in \Gamma_{n}\right\} \cup\{0\}$ is weakly compact.

Proof. It is clear that only the implication (i) $\Rightarrow$ (ii) requires proof. For this we consider the partition $\Gamma_{n}, n \geq 1$, of the set $\Gamma$ resulting from Theorem 2.2. It is easy to see that the family $\left\{x_{\gamma}: \gamma \in \Gamma_{n}\right\}$ does not contain an $\ell^{1}$-sequence and hence it is a weakly relatively compact set.

Most of the remaining part of this section is devoted to the construction of a WCG Banach space $X$ with an unconditional basis that contains a non-WCG subspace also having an unconditional basis. The space $X$ will be a direct sum, in the $\ell^{2}$-norm of a sequence $X_{n}, n \geq 1$, of WCG Banach spaces each with an unconditional basis. The definition of the spaces $X_{n}, n \geq 1$, is based on an example of a compact space given by Talagrand ([T], Th. 4.3) and on a rather standard method of construction of Banach spaces having an unconditional basis, which can be found for instance in $\mathrm{AM}$ (Def. 3.13 and 2.1 (iii)). We briefly describe the method. 
We recall that a family $\mathcal{A}$ of subsets of a nonempty set $\Gamma$ is said to be adequate if it satisfies the following properties:

(i) $\{\gamma\} \in \mathcal{A}$, for all $\gamma \in \Gamma$,

(ii) $A \subseteq B \in \mathcal{A} \Rightarrow A \in \mathcal{A}$ and

(iii) if $A \subseteq \Gamma$ such that every finite subset of $A$ belongs to $\mathcal{A}$, then $A \in \mathcal{A}$.

Note that if $\mathcal{A}$ is an adequate family of subsets of $\Gamma$, then the space $K \equiv K(\mathcal{A})=$ $\left\{\chi_{A}: A \in \mathcal{A}\right\}$ is a compact subspace of the compact space $\{0,1\}^{\Gamma}$.

Let $\mathcal{A}$ be an adequate family of subsets of the set $\Gamma$; then we can define, by using $\mathcal{A}$, a Banach space $X \equiv X_{1,2}(\mathcal{A})$ in the following way: $X$ will be the completion of the space $c_{00}(\Gamma)$ of finitely supported real functions on $\Gamma$, under the norm

$$
\|f\|=\sup \left\{\left(\sum_{i=1}^{n}\left(\sum_{\gamma \in A_{i}}|f(\gamma)|\right)^{2}\right)^{1 / 2}: A_{i} \cap A_{j}=\emptyset, i \neq j \text { and } A_{i} \in \mathcal{A}\right\} .
$$

It is easy to verify that the usual basis of $c_{00}(\Gamma)$, i.e. the family $\left\{e_{\gamma}: \gamma \in \Gamma\right\}$ is an unconditional normalized (boundedly complete) basis for the Banach space $X$ (see [AM], Def. 3.13).

We define now the example of the compact space of Talagrand mentioned above. In order to do this we need some terminology and notation. We denote by $\Sigma$ the set $\mathbb{N}^{\mathbb{N}}$ of sequences of positive integers, endowed with the product topology (where $\mathbb{N}$ has the discrete topology), and by $S$ the set of finite sequences of positive integers.

If $\sigma \in \Sigma$ and $n \in \mathbb{N}$, then $\sigma \mid n$ is the finite sequence $(\sigma(1), \ldots, \sigma(n))$; for $s \in S$, $s=\left(k_{1}, \ldots, k_{n}\right)$ and $\sigma \in \Sigma$, the notation $s<\sigma$ means that $\sigma \mid n=s$. It is clear that the countable family $\left\{V_{s}, s \in S\right\}$, where $V_{s}=\{\sigma \in \Sigma: s<\sigma\}$ for $s \in S$, is a basis for the topology of $\Sigma$ consisting of open and closed sets.

For $m \in \mathbb{N}$, let

$$
\mathcal{A}_{m}=\{A \subseteq \Sigma: \sigma, \tau \in A, \sigma \neq \tau \text { implies } \sigma|m=\tau| m \text { and } \sigma(m+1) \neq \tau(m+1)\} .
$$

Thus every $A \in \mathcal{A}_{m}$ is at most countable. It is easily verified that:

(a) $\mathcal{A}_{m}$ is adequate for all $m \in \mathbb{N}$,

(b) $\mathcal{A}_{m} \cap \mathcal{A}_{n}=\{\{\sigma\}: \sigma \in \Sigma\}$, for $m \neq n$, and

(c) $\mathcal{A}=\bigcup_{m=1}^{\infty} \mathcal{A}_{m}$ is also adequate.

Note. The compact space

$$
K \equiv K(\mathcal{A})=\left\{\chi_{A}: A \in \mathcal{A}\right\}
$$

is the first example of a compact space so that $C(K)$ is $\mathcal{K}$-analytic in its weak topology (that is, $K$ is a Talagrand compact) without being a WCG Banach space (that is, $K$ is not an Eberlein compact). The space $K$ was constructed by Talagrand in T] (Th. 4.3).

The following lemma is due to Talagrand. Its proof is an easy application of Baire's category theorem (see $[\mathrm{T}]$ and $[\mathrm{F}]$, Lemma 1.6.1).

Lemma 2.4. Let $\left(\Gamma_{n}\right), n \geq 1$, be a sequence of subsets of $\Sigma$ such that $\Sigma=\bigcup_{n=1}^{\infty} \Gamma_{n}$. Then there exist $n_{0}, m_{0} \in \mathbb{N}$ and an infinite member $A$ of $\mathcal{A}_{m_{0}}$ such that $A \subseteq \Gamma_{n_{0}}$.

Now we are coming to the construction of the desired WCG Banach space $X$. We set for every $m \in \mathbb{N}, X_{m}=X_{1,2}\left(\mathcal{A}_{m}\right)$.

Proposition 2.5. For every $m \in \mathbb{N}, X_{m}$ is a WCG Banach space with an unconditional basis. 
Proof. It is clear from the previous remarks that the family $\left\{e_{\sigma}: \sigma \in \Sigma\right\}$ is an unconditional basis for $X_{m}$, for every $m \in \mathbb{N}$. Fix any $m \in \mathbb{N}$. It is clear that

$$
\left\{e_{\sigma}: \sigma \in \Sigma\right\}=\bigcup_{|s|=m+1}\left\{e_{\sigma} \in V_{s}\right\}
$$

(where, for $s \in S,|s|$ denotes the length of $s$ ).

Let $A \in \mathcal{A}_{m}$ and $s \in S$ with $|s|=m+1$. Then obviously $\left|A \cap V_{s}\right| \leq 1$ and therefore we get, from the definition of the norm of the space $X_{m}$ that the complemented subspace $Y_{s}=\overline{\operatorname{span}}\left\{e_{\sigma}: \sigma \in V_{s}\right\}$ of $X_{m}$ is isometric with a Hilbert space, and the family $\left\{e_{\sigma}: \sigma \in V_{s}\right\}$ is the canonical basis for $Y_{s}$. It follows in particular that each member of the countable family of sets $\left\{e_{\sigma}: \sigma \in V_{s}\right\} \cup\{0\}, s \in S,|s|=m+1$, is weakly compact in $X_{m}$, so we get from (1) that $X$ is WCG.

The proof of the proposition is complete.

Now we set $X=\left(\sum_{m=1}^{\infty} \oplus X_{1,2}\left(\Sigma \times\{m\}, \mathcal{A}_{m}\right)_{2}\right.$, i.e. $X$ is equal to the direct sum, in the $\ell^{2}$-norm, of the Banach spaces $X_{m}, m \geq 1$ defined above.

Theorem 2.6. The space $X$ is $W C G$ with an unconditional basis containing a non-WCG closed linear subspace $Y$ that also has an unconditional basis.

Proof. The Banach space $X$ is WCG as a direst sum of WCG Banach spaces in the $\ell^{2}$-norm. It is also clear that the family $\left\{e_{(\sigma, m)}: \sigma \in \Sigma, m \in \mathbb{N}\right\}$ is an unconditional normalized basis for $X$. Now we are going to define the non-WCG subspace $Y$ of $X$.

So let $x_{\sigma}=\sum_{m=1}^{\infty} \frac{1}{2^{m / 2}} \cdot e_{(\sigma, m)}$, for $\sigma \in \Sigma$; then clearly $\left\|x_{\sigma}\right\|=1$ for every $\sigma \in \Sigma$. We also set $Y=\overline{\operatorname{span}}\left\{x_{\sigma}: \sigma \in \Sigma\right\}$ (= the closed linear span of the vectors $x_{\sigma}, \sigma \in \Sigma$ in $X$ ). We shall show that $Y$ is the desired subspace of $X$. The family $\left\{x_{\sigma}: \sigma \in \Sigma\right\}$ is an unconditional basis of $Y$ as a block basis of the unconditional basis $\left\{e_{(\sigma, m)}: \sigma \in \Sigma, m \in \mathbb{N}\right\}$ of $X$. Assume for the purpose of contradiction that $Y$ is WCG. Then Theorem 2.3 yields that there exists a sequence $\Gamma_{n}, n \geq 1$, of subsets of $\Sigma$ with $\Sigma=\bigcup_{n=1}^{\infty} \Gamma_{n}$ so that each set $\left\{x_{\sigma}: \sigma \in \Gamma_{n}\right\} \cup\{0\}, n \in \mathbb{N}$, is weakly compact in $Y$. Also by Lemma 2.4 there exist $m_{0}, n_{0} \in \mathbb{N}$ and an infinite member $A$ of $\mathcal{A}_{m_{0}}$ with $A \subseteq \Gamma_{n_{0}}$. Let $A=\left\{\sigma_{k}: k \in \mathbb{N}\right\}$ be an enumeration of $A$. Then clearly we have that

$$
x_{\sigma_{k}} \stackrel{w}{\rightarrow} 0 .
$$

We consider the series $\sum_{k=1}^{\infty} e_{\left(\sigma_{k}, m_{0}\right)}^{*}$. Since the subspace $\overline{\operatorname{span}}\left\{e_{\left(\sigma_{k}, m_{0}\right)}: k \in \mathbb{N}\right\}$ of $X$ is isometric with the space $\ell^{1}(\mathbb{N})$, this series defines an element $x^{*} \in X^{*}$. Let $\lambda$ be any positive integer. Then we have $x^{*}\left(x_{\sigma_{\lambda}}\right)=\left(\sum_{k=1}^{\infty} e_{\left(\sigma_{k}, m_{0}\right)}^{*}\right)\left(\sum_{m=1}^{\infty} \frac{1}{2^{m / 2}} e_{\left(\sigma_{\lambda}, m\right)}\right)$ $=e_{\left(\sigma_{\lambda}, m_{0}\right)}^{*}\left(\frac{1}{2^{m_{0} / 2}} \cdot e_{\left(\sigma_{\lambda}, m_{0}\right)}\right)=\frac{1}{2^{m_{0} / 2}}$, which contradicts $(1)$. The proof of the theorem is complete.

It is well-known that any WCG Banach space $X$ admits an $M$-basis $\left\{\left(x_{\gamma}, f_{\gamma}\right)\right.$ : $\gamma \in \Gamma\}$ so that the set $\left\{x_{\gamma}: \gamma \in \Gamma\right\} \cup\{0\}$ is weakly compact (see [HHZ]). It is natural to ask whether any $M$-basis of a WCG Banach space satisfies Johnson's property (i.e. there is a partition $\left(\Gamma_{n}\right)_{n}$ of $\Gamma$ such that $\left\{x_{\gamma}: \gamma \in \Gamma_{n}\right\}$ is weakly relatively compact for all $n \in \mathbb{N}$ ). With the next result we shall provide an example of an $M$-basis that fails Johnson's property. Additionally this example shows that the conclusion of Theorem $[2.2$ is in a sense the best possible. 
Proposition 2.7. The WCG Banach space $X$ of Theorem 2.6] admits an $M$-basis that cannot be written as a countable union of weakly relatively compact sets.

Proof. For every $\sigma \in \Sigma$ we consider the subspace, $X_{\sigma}=\overline{\operatorname{span}}\left\{e_{(\sigma, m)}: m \geq 1\right\}$ of $X$; it is clear that each $X_{\sigma}$ is isometric to a separable Hilbert space having the set $\left\{e_{(\sigma, m)}: m \in \mathbb{N}\right\}$ as an unconditional basis. It is also clear that $X=\sum_{\sigma \in \Sigma} \oplus X_{\sigma}$. By making a Gram-Schmidt orthonormalization, we can find for every $\sigma \in \Sigma$ an orthonormal basis $\left\{x_{\sigma}^{n}: n \geq 1\right\}$ of $X_{\sigma}$ so that $x_{\sigma}^{1}=x_{\sigma}\left(=\sum_{m=1}^{\infty} \frac{1}{2^{m / 2}} e_{(\sigma, m)}\right)$. Now we set

$$
f_{\sigma}^{n}=\left\{\begin{array}{l}
x_{\sigma}^{n}, n \geq 2, \\
\sum_{m=1}^{\infty} \frac{1}{2^{m / 2}} e_{(\sigma, m)}^{*}, n=1,
\end{array}\right.
$$

for every $\sigma \in \Sigma$ and $n \in \mathbb{N}$ and claim that the family $\left\{\left(x_{\sigma}^{n}, f_{\sigma}^{n}\right): \sigma \in \Sigma, n \geq 1\right\}$ is an $M$-basis for $X$ that satisfies the desired property. Clearly the second part of our claim requires justification. So let us assume that the set $\left\{x_{\sigma}^{n}: \sigma \in \Sigma, n \geq 1\right\}$ is equal to a countable union of weakly relatively compact sets. Then since (obviously) the unconditional basis $\left\{x_{\sigma}: \sigma \in \Sigma\right\}$ of the space $Y$ is contained in the set $\left\{x_{\sigma}^{n}\right.$ : $\sigma \in \Sigma, n \geq 1\}$ we would have that the space $Y$ is WCG, a contradiction.

Remark 2.8. Observe that for every $\sigma \in \Sigma$ the sequence $\left\{e_{(\sigma, m)}: m \in \mathbb{N}\right\}$ is isometric to the usual basis of $\ell^{2}(\mathbb{N})$. Also for a given $\sigma$ the set $\left\{x_{\sigma}^{n}: n \geq 1\right\}$ is also an orthonormal basis for the space $X_{\sigma}=\overline{\operatorname{span}}\left\{e_{(\sigma, m)}: m \in \mathbb{N}\right\}$. Therefore the following peculiar phenomenon occurs. In a space with an unconditional basis $\left(e_{(\sigma, m)}\right)_{\sigma \in \Sigma, m \in \mathbb{N}}$ we decompose the basis into a family $\left\{F_{\sigma}=\left\{e_{(\sigma, m)}\right\}_{m \in \mathbb{N}}\right\}_{\sigma \in \Sigma}$ of pairwise disjoint sets, and in the space $X_{\sigma}$ we select a new unconditional basis $\left(x_{\sigma}^{n}\right)_{n}$ such that the new biorthogonal system $\left\{x_{\sigma}^{n}\right\}_{\sigma \in \Sigma, n \in \mathbb{N}}$ is not an unconditional basis. However we can split this system into countably many pieces (i.e., $Q_{n}=\left\{x_{\sigma}^{n}\right\}_{\sigma \in \Sigma}$, each one being an unconditional basis.

Summing up all the previous results we have the following.

Theorem 2.9. There exists a $W C G$ space $X$ with an unconditional basis $\left(e_{a}\right)_{a \in \mathcal{A}}$ such that

(i) There exists a countable partition of $\mathcal{A}$ into $\left(\mathcal{A}_{n}\right)_{n \in \mathbb{N}}$ such that $\left(e_{a}\right)_{a \in \mathcal{A}_{n}}$ is isometric to the usual basis of $\ell^{2}\left(\mathcal{A}_{n}\right)$.

(ii) There exists an $M$-basis $\left(x_{\gamma}\right)_{\gamma \in \Gamma}$ of $X$ not admitting a countable partition with each member being a weakly relatively compact set.

(iii) For this $M$-basis there exists a partition $\left(x_{\gamma}\right)_{\gamma \in \Gamma_{n}}, n \in \mathbb{N}$, such that for each $n \in \mathbb{N}$ the space $X_{n}=\overline{\operatorname{span}}\left\{\left(x_{\gamma}\right)_{\gamma \in \Gamma_{n}}\right\}$ is not $W C G$ and $\left(x_{\gamma}\right)_{\gamma \in \Gamma_{n}}$ is a 1-unconditional basis for the space $X_{n}$.

Proof. (i) The desired space $X$ is the space defined above, namely, $X=$ $\left(\sum_{m} \oplus X_{m}\right)_{2}$. The unconditional basis is the family $\left(e_{(\sigma, n)}\right)_{\sigma \in \Sigma, n \in \mathbb{N}}$. As in the proof of Proposition 2.5 we decompose the set $\Sigma$ into the families $\left\{V_{s}:|s|=n+1\right\}$. Each family $\left\{e_{(\sigma, n)}: \sigma \in V_{s}\right\}$ is isometric to the usual basis of $\ell^{2}\left(V_{s}\right)$, and the families $\left\{e_{(\sigma, n)}: \sigma \in V_{s}\right\},|s|=n+1, n \in \mathbb{N}$, define a countable partition of the basis of $X$.

(ii) It follows from Proposition 2.7 .

(iii) The proof of Proposition 2.7 yields that $x_{\sigma}^{1}=\sum_{m} \frac{1}{2^{m / 2}} e_{(\sigma, m)}$ and also for $n \geq 2$,

$$
x_{\sigma}^{n}=\lambda_{n}\left(\mu_{1} e_{(\sigma, 1)}+\cdots+\mu_{n-1} e_{(\sigma, n-1)}+\sum_{m \geq n} \frac{1}{2^{m / 2}} e_{(\sigma, m)} .\right.
$$


Hence using similar arguments to the proof of the fact that $\left(x_{\sigma} \equiv x_{\sigma}^{1}\right)_{\sigma \in \Sigma}$ generates a non-WCG subspace of $X$, we obtain the same property for every family $\left(x_{\sigma}^{n}\right)_{\sigma \in \Sigma}$. The proof of the theorem is complete.

\section{SEQUenCES OF TREES}

A tree is a partially ordered set $(T, \leq)$ such that $\{s \in T: s \leq t\}$ is a well-ordered subset of $T$ for all $t \in T$.

Let $(T, \leq)$ be any tree. A chain in $(T, \leq)$ is any totally ordered (and thus wellordered) subset of $(T, \leq)$. Any maximal chain is called a branch of $(T, \leq)$. A segment or interval $I$ of $(T, \leq)$ is any chain so that, $s<t<u$ and $s, u \in I$ imply that $t \in I$. An element $t$ of $T$ is called an immediate successor of $s \in T$ if $s<t$ and there is no $x \in T$ so that $s<x<t$.

For every $t \in T$, we set $S_{t}=\{u \in T: u$ is an immediate successor of $t\}$, $T_{t}=\{u \in T: t \leq u\}$ and $I_{t}=\{s \in T: s \leq t\}$ ( $I_{t}$ is called an initial segment of $(T, \leq))$.

We denote by $o(t)$ for $t \in T$ the order type of $I_{t} \backslash\{t\}=\{s \in T: s<t\}$. If $\alpha$ is any ordinal, then the $\alpha$-th level of $T$, or $\operatorname{Lev}_{\alpha}(T)$ is the set $\{t \in T: o(t)=\alpha\}$. The height of $T$, or $h t(T)$ is the least $\alpha$ such that $\operatorname{Lev}_{\alpha}(T)=\emptyset$; clearly $\operatorname{Lev}_{0}(T)$ is the set of minimal elements of $T$. If $\operatorname{Lev}_{0}(T)=\{t\}$, then we say that $t$ is the root of $T$.

Definition 3.1. A sequence $T_{n}, n \geq 1$, of trees will be called a Rezničenko sequence of trees if:

(i) $\left|\operatorname{Lev}_{0}\left(T_{n}\right)\right|=1$ for all $n \geq 1$ and $\operatorname{Lev}_{0}\left(T_{n}\right) \cap \operatorname{Lev}_{0}\left(T_{m}\right)=\emptyset$, for $m \neq n$.

(ii) $h t\left(T_{n}\right)=\omega$ for all $n \geq 1$ and for every $t \in T_{n}$ the set $S_{t}$ is uncountable.

(iii) For every $I_{1}, I_{2}$ segments of $T_{n_{1}}, T_{n_{2}}$ respectively, $n_{1} \neq n_{2}$, we have that $\left|I_{1} \cap I_{2}\right| \leq 1$.

(iv) For every non-empty subset $M$ of $\mathbb{N}, I_{n}$ initial segments of $T_{n}, n \in M$, such that $I_{n} \cap I_{m}=\emptyset$ for $m \neq n$, there exist uncountably many $t \in T=\bigcup_{n=1}^{\infty} T_{n}$ so that $t \in S_{\max I_{n}}$ for all $n \in M$.

We refer to $[\mathrm{A}]$ and $[\mathrm{F}$ for proofs of the existence of a sequence of Rezničenko trees. But for the convenience of the reader we give below a sketch of the proof of the existence of such a sequence of trees.

Proposition 3.2. There exists a sequence of Rezničenko trees.

An outline of the proof. Every tree $T_{n}, n \in \mathbb{N}$, will be realized as $\bigcup_{\xi<\omega_{1}} T_{n}^{\xi}$ with $T_{n}^{\xi}, \xi<\omega_{1}, n \in \mathbb{N}$, satisfying the following inductive properties:

(i) $T_{n}^{\xi}$ is a tree, $h t\left(T_{n}^{\xi}\right) \leq \omega, T_{n}^{\xi} \subseteq[0, \xi] \times 2^{\omega}$ and $(0, n)$ is the root of $T_{n}^{\xi}$ (where $2^{\omega}$ is considered as an ordinal number).

(ii) For $\zeta<\xi<\omega_{1}, T_{n}^{\zeta}$ is a subtree of $T_{n}^{\xi}$ (especially here by the term subtree we mean that, if $t \in T_{n}^{\zeta}$, then $\left\{s \in T_{n}^{\xi}: s<t\right\} \subseteq T_{n}^{\zeta}$ ).

(iii) If $\xi=\zeta+1, M$ is a non-empty subset of $\mathbb{N}$ and, for $n \in M, I_{n}$ is an initial segment of $T_{n}^{\zeta}$ such that $I_{n} \cap I_{m}=\emptyset$ for $m \neq n$, then the set $\left\{t \in 2^{\omega}:(\xi, t)\right.$ is an immediate successor of $\max I_{n}$ for all $\left.n \in M\right\}$ is uncountable.

(iv) If $I_{1}, I_{2}$ are initial segments of $T_{n_{1}}^{\xi}, T_{n_{2}}^{\xi}, n_{1} \neq n_{2}$, then $\left|I_{1} \cap I_{2}\right| \leq 1$.

We proceed inductively and set $T_{n}^{0}=\{(0, n)\}$ for $n \geq 1$. Let $\xi<\omega_{1}$ and assume that $T_{n}^{\zeta}$ have been constructed for all $\zeta<\xi$ and $n \in \mathbb{N}$.

If $\xi$ is a limit ordinal, we set $T_{n}^{\xi}=\bigcup_{\zeta<\xi} T_{n}^{\zeta}$, for $n \in \mathbb{N}$. If $\xi=\zeta+1$, we consider all sets of the form $K=\left\{I_{n}: n \in M\right\}$ where $M$ is any non-empty subset of $\mathbb{N}, I_{n}$ 
is an initial segment of $T_{n}^{\zeta}$ for $n \in M$ and $I_{n} \cap I_{m}=\emptyset$ for $m \neq n$. The cardinality of all such $K$ is $2^{\omega}$, and we order them as $\left\{K_{t}^{\xi}: t<2^{\omega}\right\}$ so that each $K$ appears uncountably many times in this enumeration. We define $T_{n}^{\xi}$, for a given $n \in \mathbb{N}$ in the following way. We set for every $(\eta, t) \in T_{n}^{\zeta},(\eta, t)_{\xi}^{+}=\left\{\left(\xi, t^{\prime}\right)\right.$ : there exists $I \in K_{t^{\prime}}^{\xi}, I \subseteq T_{n}^{\zeta}$ and $\left.\max I=(\eta, t)\right\}$. Then we set

$$
T_{n}^{\xi}=T_{n}^{\zeta} \cup\left\{(\eta, t)_{\xi}^{+}:(\eta, t) \in T_{n}^{\zeta}\right\}
$$

and order $T_{n}^{\xi}$ in the obvious way.

The inductive construction is complete. It is clear that the inductive properties (i) to (iv) stated above imply that $T_{n}, n \geq 1$, is a sequence of Rezničenko trees.

Note. It can be shown that the compact space $K=\left\{\chi_{I}: I\right.$ is a segment of $T_{n}$, for some $n \in \mathbb{N}\} \cup\{0\}$ is still another example of a Talagrand compact space that is not an Eberlein compact space (cf. $\underline{\underline{A}},[\underline{\mathrm{F}}]$ ).

In the sequel we prove a Baire category type property of sequences of Rezničenko trees.

Proposition 3.3. Let $T_{n}, n \geq 1$, be a sequence of Rezničenko trees, and let $T=$ $\bigcup_{n=1}^{\infty} T_{n}$. If $T=\bigcup_{k=1}^{\infty} D_{k}$, then there exists $k_{0} \in \mathbb{N}$ such that the set $D_{k_{0}} \cap T_{k_{0}}$ contains an infinite segment of the tree $T_{k_{0}}$.

Proof. We first make the following

Claim. There exists $k_{0} \in \mathbb{N}$ and $a_{0} \in T_{k_{0}}$ such that for every $\beta \in T_{k_{0}}^{a_{0}}=\left\{\beta \in T_{k_{0}}\right.$ : $\left.a_{0} \leq \beta\right\}$ we have $\left|S_{\beta} \cap D_{k_{0}}\right| \geq \omega_{1}$.

Proof of the Claim. Assume for the purpose of contradiction that the claim does not hold. Therefore for every $k \in \mathbb{N}$ and every $a \in T_{k}$ there exists $\beta \in T_{k}^{a}$ such that $\left|S_{\beta} \cap D_{k}\right| \leq \omega$. We observe that for each $k \in \mathbb{N}$ and every finite subset $F$ of $T_{k}$, not containing the root of $T_{k}$, there exists $a \in T_{k}$ such that $T_{k}^{a} \cap F=\emptyset$ and also $\{\beta: \beta \leq a\} \cap F=\emptyset$. Therefore proceeding by induction we construct a sequence of pairwise disjoint initial segments $I_{\beta_{k}}, k \in \mathbb{N}$, respectively, such that $\left|S_{\beta_{k}} \cap D_{k}\right| \leq \omega$ for all $k \in \mathbb{N}$.

Since by property (iv) of the definition of Rezničenko trees (Definition 3.1) the set $\bigcap_{k=1}^{\infty} S_{\beta_{k}}$ is uncountable, there exists $n \in \mathbb{N}$ such that $\left|\bigcap_{k=1}^{\infty} S_{\beta_{k}} \cap D_{n}\right| \geq \omega_{1}$, a contradiction.

Now let $k_{0}$ be the natural number given by the claim. Then it is easy to construct by repeated use of the claim the desired infinite segment (beginning from $a_{0}$ ) in the set $D_{k_{0}} \cap T_{k_{0}}$.

\section{The HeREDITY PROBLEM FOR SPACES NOT CONTAINING $\ell^{1}$}

Given any tree $(T, \leq)$, a James space denoted by $J(T)$ is defined as follows: $J(T)$ is the completion of the linear space $c_{00}(T)$ under the norm ("James-norm")

$$
\|f\|=\sup \left\{\left(\sum_{i=1}^{n}\left(\sum_{t \in S_{i}} f(t)\right)^{2}\right)^{1 / 2}: S_{1}, \ldots, S_{n}\right. \text { are pairwise }
$$

$$
\text { disjoint segments of }(T, \leq)\}
$$

(see [HO]). 
We note the following easily verified facts:

(i) If $S$ is a nonempty subset of $(T, \leq)$, then $S$ is also a tree with the induced order from $(T, \leq)$ and the Banach space $J(S)$ is isometrically embedded in the space $J(T)$. In particular, if $(S, \leq)$ has the property $\{s \in T: s<t\} \subseteq S$ for every $t \in S$, then the space $J(S)$ is complemented in $J(T)$.

(ii) Given any $\xi \leq h t(T)$ let us denote, for convenience, by $T(\xi)$ the $\xi$-th level of $(T, \leq)$ (i.e., the set $\left.\operatorname{Lev}_{\xi}(T)\right)$ and by $T_{\xi}$ the union $\bigcup_{\zeta<\xi} T(\zeta+1)$. Then the natural map $P_{\xi}: J(T) \rightarrow J\left(T_{\xi}\right):\left.f \rightarrow f\right|_{T_{\xi}}$ is a norm one projection (see (i) above).

(iii) If we denote by $(D, \leq)$ the dyadic tree (i.e., that tree with a unique root, $h t(D)=\omega$ and so that each element of $(D, \leq)$ has exactly two immediate successors), then every countable tree $(S, \leq)$ with $h t(S) \leq \omega$ is order isomorphic to a subtree of $(D, \leq)$.

Note. The James tree space is the James space $J(D)$, where $(D, \leq)$ is the dyadic tree. It is well-known that $J(D)$ is $\ell^{2}$-saturated, i.e. every infinite-dimensional subspace of $J(D)$ contains a further subspace isomorphic to the Hilbert space $\ell^{2}(\mathbb{N})$ (see [J] and [HO]). Using this fact, Hagler and Odell proved in [HO that this is a general phenomenon for the class of James-type Banach spaces, $J(T)$, where $(T, \leq)$ is any tree. For the sake of completeness we give below a move extensive description of the involving part of their argument.

Theorem 4.1 (Hagler - Odell). Let $(T, \leq)$ be a tree. Then every infinite-dimensional subspace $Y$ of $J(T)$ contains an infinite-dimensional subspace $Z$ isomoprhic to a subspace of $J(D)$ (and hence by the above remarks $J(T)$ is $\ell^{2}$-saturated).

Proof. Let $\xi$ be the least ordinal $\zeta \leq h t(T)$ such that the operator $P_{\zeta}: Y \rightarrow J\left(T_{\zeta}\right)$ is not strictly singular (i.e., there exists an infinite-dimensional subspace $Y_{1}$ of $Y$ so that $\left.P_{\zeta}\right|_{Y_{1}}$ is an isomorphic embedding). Let $Z_{0}$ be an infinite-dimensional subspace of $Y$ so that $P_{\xi}: Z_{0} \rightarrow J\left(T_{\xi}\right)$ is an isomorphic embedding. Hence there exists $\theta>0$ such that $\left\|P_{\xi}(z)\right\| \geq \theta$ for every $z \in Z_{0}$ with $\|z\|=1$. We distinguish two cases:

(I) $\xi$ is a successor ordinal, say $\xi=\zeta+1$. Since the operator $P_{\zeta}: Y \rightarrow J\left(T_{\zeta}\right)$ is strictly singular, for every infinite-dimensional subspace $Z$ of $Y$ and each $\varepsilon>0$ there exists $z_{\varepsilon} \in Z,\left\|z_{\varepsilon}\right\|=1$ with $\left\|P_{\zeta}\left(z_{\varepsilon}\right)\right\|<\varepsilon$. Let $\varepsilon_{1} \in(0, \theta)$, pick $z_{1} \in Z_{0}$, $\left\|z_{1}\right\|=1$ so that $\left\|P_{\zeta}\left(z_{1}\right)\right\|<\varepsilon_{1}$. Thus $\left\|P_{\zeta+1}\left(z_{1}\right)\right\| \geq \theta$ and $\left\|\left(P_{\zeta+1}-P_{\zeta}\right)\left(z_{1}\right)\right\| \geq$ $\theta-\varepsilon_{1}$. We choose a finite subset $F_{1}$ of the level $T(\zeta+1)$ of $(T, \leq)$ and a vector $w_{1} \in\left\langle e_{a}: a \in F_{1}\right\rangle$ such that $\left\|P_{\zeta+1}\left(z_{1}\right)-w_{1}\right\|<\varepsilon_{1}$. Note that $\left\|w_{1}\right\| \geq \theta-\varepsilon_{1}$. We set $Z_{1}=\left\{z \in Z_{0}: e_{a}^{*}(z)=0\right.$, for $\left.a \in F_{1}\right\}$ and note that $Z_{1}$ is infinite-dimensional. We proceed by induction and for an appropriate decreasing sequence $\left(\varepsilon_{n}\right) \subseteq(0, \theta)$, choose a sequence $\left(z_{n}\right) \subseteq Z_{0}$, a sequence $\left(w_{n}\right) \subseteq J(T)$ supported on a sequence of finite pairwise disjoint sets $\left(F_{n}\right)$ with $\bigcup_{n=1}^{\infty} F_{n} \subseteq T(\zeta+1)$ so that $\left\|z_{n}\right\|=1$, $\left\|P_{\zeta+1}\left(z_{n}\right)-w_{n}\right\| \leq \varepsilon_{n}$ and $\left\|w_{n}\right\| \geq \theta-\varepsilon_{n}$ for $n \geq 1$. The sequence $\left(w_{n}\right)$ is clearly equivalent to the canonical basis of $\ell^{2}(\mathbb{N})$, and if the sum $\sum_{n=1}^{\infty} \varepsilon_{n}$ is sufficiently small, then the sequence $\left(P_{\zeta+1}\left(z_{n}\right)\right)$ is equivalent to $\left(w_{n}\right)$. So we are done in the case when $\xi$ is a successor ordinal.

(II). Assume that $\xi$ is a limit ordinal. Let $z_{1} \in Z_{0},\left\|z_{1}\right\|=z$, thus $\left\|P_{\xi}\left(z_{1}\right)\right\| \geq \theta$. Let $\varepsilon_{1} \in(0, \theta)$, pick a finite subset $F_{1} \subseteq T_{\xi}$ and $w_{1} \in\left\langle e_{a}: a \in F_{1}\right\rangle$ such that $\left\|P_{\xi}\left(z_{1}\right)-w_{1}\right\|<\varepsilon_{1}$, hence $\left\|w_{1}\right\| \geq \theta-\varepsilon_{1}$. Set $\zeta_{1}=\max \left\{o(a): a \in F_{1}\right\}$, clearly $\zeta_{1}<\xi$. Let again $\left(\varepsilon_{n}\right)$ be an appropriate sequence in the interval $(0, \theta)$. We 
construct by induction sequences $\left(z_{n}\right) \subseteq Z_{0},\left(w_{n}\right) \subseteq J(T)$ and a sequence of ordinals $\zeta_{1}<\zeta_{2}<\ldots<\zeta_{n}<\ldots<\xi$ such that

(a) each $w_{n}$ is supported on a finite set $F_{n}$ such $F_{1} \subseteq T_{\zeta_{1}}$ and $F_{n} \subseteq T_{\xi} \backslash T_{\zeta_{n-1}}$ for $n \geq 2$,

(b) $\left\|z_{n}\right\|=1$ and $\left\|P_{\xi}\left(z_{n}\right)-w_{n}\right\|<\varepsilon_{n}$, for $n \geq 1$.

The tree $F=\bigcup_{n=1}^{\infty} F_{n}$ (with the induced order from $(T, \leq)$ ) is clearly countable and $h t(F)=\omega$. Therefore, by facts (i) and (ii) the James space $J(F)$ is embedded into the space $J(D)$. Since the James tree space $J(D)$ is $\ell^{2}$-saturated its subspace $X$, generated by the basic sequence $\left(w_{n}\right)_{n \geq 2}$, contains isomorphically the space $\ell^{2}(\mathbb{N})$. On the other hand (b) implies that (if the sum $\sum_{n=1}^{\infty} \varepsilon_{n}$ is quite small) $X$ is isomorphic to the space $\overline{\operatorname{span}}\left\{P_{\xi}\left(z_{n}\right): n \geq 1\right\}$, which is embedded into $Z_{0}$ and thus into $Y$.

In the following theorem some properties of James spaces $J(T)$ on trees $(T, \leq)$ of height $\omega$ are stated. We give an outline of the proof of this theorem.

Theorem 4.2. Let $(T, \leq)$ be a tree of height $\omega(h t(T)=\omega)$ and set $X=J(T)$. Then we have

(i) the Banach space $X$ is $W C G$;

(ii) $X^{* *} \cong X \oplus \ell^{2}\left(\mathcal{B}_{T}\right)$, where $\mathcal{B}_{T}$ is the set of (infinite) branches of $(T, \leq$ ) (if $(T, \leq)$ has no infinite branch, then $X$ is reflexive), and hence in particular $X^{* *}$ is $W C G$ and does not contain $\ell^{1}$.

Proof. (i) It is easy to verify that if $T(n)$ is the $n$-th level $(n \in \mathbb{N})$ of $(T, \leq)$, then the set $\left\{e_{t}: t \in T(n)\right\}$ is isometrically equivalent to the canonical basis of the space $\ell^{2}(T(n))$. Thus each set $\left\{e_{t}: t \in T(n)\right\} \cup\{0\}$ is weakly compact in $X$. Hence $X$ is WCG.

(ii) For any nonempty segment $S$ the functional $S^{*}$ is defined as

$$
S^{*}(x)=\sum_{t \in S} x(t),
$$

and it is easy to verify that $\left\|S^{*}\right\|=1$. Consider the set

$$
\begin{aligned}
& D=\left\{\sum_{i=1}^{n} \lambda_{i} S_{i}^{*}: \lambda_{i} \in \mathbb{R},\left\{S_{i}\right\}_{i=1}^{n} \text { pairwise disjoint segments of }(T, \leq)\right. \\
& \text { and } \left.\sum_{i=1}^{n} \lambda_{i}^{2} \leq 1\right\} .
\end{aligned}
$$

It is easy to verify that $D$ is a 1-norming set for $X$ and also that it is norm dense in its $w^{*}$-closure. Since $\ell^{1}$ does not embed in $X$, Haydon's classical theorem $[\mathrm{H}]$ yields that $D$ generates in norm the space $X^{*}$. Thus we conclude that the set $\left\{S^{*}: S\right.$ is a segment of $\left.T\right\}$ generates in norm the space $X^{*}$ (e.g. $[\mathrm{A}],[\mathrm{F}]$ ).

Let $Z$ be the closed linear span of the set $\left\{e_{t}^{*}: t \in T\right\}$ in $X^{*}$. Then it can be shown as in the case of the dyadic tree $(D, \leq)$ (e.g. [LS]) that $Z$ is a predual of $X$ and $X^{*} / Z \cong \ell^{2}\left(\mathcal{B}_{T}\right)$ (if there is no infinite branch of $(T, \leq)$, i.e., if $\mathcal{B}_{T}=\emptyset$, then clearly $Z=X^{*}$ and the space $X$ is reflexive). Assume that $\mathcal{B}_{T} \neq \emptyset$. Then we get that $X^{* *} \cong X \oplus \ell^{2}\left(\mathcal{B}_{T}\right)$. Thus the space $X^{* *}$ is WCG as a direct sum of two WCG spaces; it is also not difficult to see that (for the same reason) $X^{* *}$ is $\ell^{2}$-saturated and hence does not contain the space $\ell^{1}$. 
Theorem 4.3. There exists a WCG Banach space $X$ such that $X^{* *}$ is also $W C G$ not containing $\ell^{1}$. Moreover there exists a closed subspace $Y$ of $X$ with the properties

(i) The spaces $Y$ and $Y^{* *}$ are not $W C G$.

(ii) The quotient $X / Y$ is a reflexive space.

Proof. We start with the definition of the space $X$. Let $T_{m}, m \geq 1$, be a sequence of Rezničenko trees. Set $X_{m}=J\left(T_{m} \times\{m\}\right)$ and $X=\left(\sum_{m=1}^{\infty} \oplus X_{m}\right)_{2}$. Since each tree $T_{m}$ is of height $\omega$, we have that each $X_{m}$ is a WCG space and also that $X_{m}^{* *} \cong X_{m} \oplus \ell^{2}\left(\mathcal{B}_{m}\right)$ (where $\mathcal{B}_{m}$ is the set of branches of $T_{m}$ ) is also a WCG space. Therefore the spaces $X$ and $X^{* *}$ are WCG and do not contain $\ell^{1}$. We pass now to define the subspace $Y$. For every $t \in T=\bigcup_{m=1}^{\infty} T_{m}$ we set $D_{t}=\left\{m \in \mathbb{N}: t \in T_{m}\right\}$ and $x_{t}=\sum_{m \in D_{t}} \frac{1}{2^{m / 2}} \cdot e_{(t, m)}$ (clearly $\left.\left\|x_{t}\right\| \leq 1\right)$. Finally we define $Y=\operatorname{span}\left\{x_{t}\right.$ : $t \in T\}$.

Claim I. There exists a family $\left\{f_{t}: t \in T\right\} \subseteq Y^{*}$ so that the family $\left\{\left(x_{t}, f_{t}\right): t \in\right.$ $T\}$ is an $M$-basis for $Y$.

Proof of Claim I. It is clear that it is enough to find a family of functionals $\left\{x_{t}^{*}\right.$ : $t \in T\} \subseteq Y^{*}$ such that $x_{t}^{*}\left(x_{t}\right) \neq 0$ for $t \in T$ and $x_{t}^{*}\left(x_{s}\right)=0$ for $t \neq s$ that separates the points of $Y$ (then we set $f_{t}=\frac{x_{t}^{*}}{x_{t}^{*}\left(x_{t}\right)}$ for $t \in T$ ). We consider the natural embedding $I: Y \rightarrow X(I(x)=x$, for $x \in Y)$ and its dual operator $I^{*}: X^{*} \rightarrow Y^{*}$. Letting $t \in T$, it is easy to verify that all functionals $\left\{I^{*}\left(e_{(t, m)}^{*}\right): m \in D_{t}\right\}$ of the space $Y$ have the same kernel, which is the subspace $Z$ of $Y$ that is generated from the vectors $x_{s}, s \in T, t \neq s$. Therefore if we normalize some one of them and denote it by $x_{t}^{*}$, we shall have that, for every $m \in D_{t}$, there exists a real number $\lambda_{(t, m)} \neq 0: I^{*}\left(e_{(t, m)}^{*}\right)=\lambda_{(t, m)} \cdot x_{t}^{*}$. It is clear that the family $\left\{x_{t}^{*}: t \in T\right\}$ satisfies the desired properties.

We note that if $t \in T$ and $m \in D_{t}$, then we have $x_{t}^{*}\left(x_{t}\right)=\frac{I^{*}\left(e_{(t, m)}^{*}\right)\left(x_{t}\right)}{\lambda_{(t, m)}}=$ $\frac{1}{2^{m / 2} \cdot \lambda_{(t, m)}}$ and thus

$$
f_{t}=\frac{x_{t}^{*}}{x_{t}^{*}\left(x_{t}\right)}=\frac{I^{*}\left(e_{(t, m)}^{*}\right)}{\lambda_{(t, m)} \cdot x_{t}^{*}\left(x_{t}\right)}=2^{m / 2} \cdot I^{*}\left(e_{(t, m)}^{*}\right) .
$$

Claim II. Let $m \in \mathbb{N}$ and let $b=\left\{t_{1}<\ldots<t_{n}<\ldots\right\}$ be any branch of the tree $T_{m}$. Then the series $\sum_{k=1}^{\infty} f_{t_{k}}$ is weak* convergent in $Y^{*}$ (and thus the sequence $\sum_{k=1}^{n} f_{t_{k}}, n \geq 1$ is norm bounded).

Proof of Claim II. It is clear that the subspace $Y_{b}=\overline{\operatorname{span}}\left\{e_{\left(t_{k}, m\right)}: k \geq 1\right\}$ of $X$ is isomorphic to the James space and complemented (in $X_{m}$ and hence) in $X$. Therefore the series $\sum_{k=1}^{\infty} e_{\left(t_{k}, m\right)}^{*}$ is weak* convergent to some $x^{*} \in X^{*}$. Therefore,

$$
2^{m / 2} \cdot I^{*}\left(x^{*}\right) \stackrel{w^{*}}{=} \sum_{k=1}^{\infty} 2^{m / 2} \cdot I^{*}\left(e_{\left(t_{k}, m\right)}^{*}\right) .
$$

Since $m \in D_{t_{k}}$, for all $k \geq 1$, we get from (2) above that $2^{m / 2} \cdot I^{*}\left(e_{\left(t_{k}, m\right)}^{*}\right)=f_{t_{k}}$, for all $k \geq 1$. It then follows immediately from (3) that the series $\sum_{k=1}^{\infty} f_{t_{k}}$ is weak* convergent in $Y^{*}$.

We are ready to prove that $Y$ is not WCG. Assume the contrary. Then by Claim I, the family $\left\{\left(x_{t}, f_{t}\right): t \in T\right\}$ is an $M$-basis for $Y$ and by Theorem [2.2 
there exists a sequence $D_{n}, n \geq 1$, of subsets of $T$ with $T=\bigcup_{n=1}^{\infty} D_{n}$ such that if a sequence $\left(t_{k}\right)_{k} \subset D_{m}$ for some $m \in \mathbb{N}$, then $\liminf _{n}\left\|\sum_{k=1}^{n} f_{t_{k}}\right\|=+\infty$. On the other hand, Proposition 3.3 yields that there exists $k_{0} \in \mathbb{N}$ with the property that the set $D_{k_{0}} \cap T_{k_{0}}$ contains an infinite segment of the tree $T_{k_{0}}$. Claim II derives a contradiction and thus $Y$ is not WCG.

For the remaining part we first observe that for the $b=\left\{t_{1}<t_{2}<\cdots<t_{n}<\right.$ $\cdots\}$ branch of $T_{m_{0}}$, the sequence $\left(e_{t_{k}}\right)_{k} w^{*}$-converges to $e_{b}^{* *} \in X_{m_{0}}^{* *}$. Also the space $X^{*}$ is norm generated by the family $\left\{S^{*}: S\right.$ is a segment of $\left.T_{m}, m \in \mathbb{N}\right\}$. We prove the following:

Claim III. For every $b=\left(t_{k}\right)_{k}$ branch of $T_{m_{0}}$ the sequence $\left(x_{t_{k}}\right)_{k} w^{*}$-converges to $\frac{1}{2^{m_{0} / 2}} e_{b}^{* *}$.

Proof of Claim III. Indeed for $m \neq m_{0}$, property (iii) of $\left(T_{n}\right)_{n}$ yields that $\left\{e_{t_{k}}\right.$ : $\left.t_{k} \in T_{m}\right\}$ is either a finite set or a weakly null sequence. Therefore for $S$ a segment of $T_{m}, m \neq m_{0}, S^{*}\left(x_{t_{k}}\right) \rightarrow 0$. Also for $S$ a segment of $T_{m_{0}}, S^{*}\left(x_{t_{k}}\right) \rightarrow \frac{1}{2^{m_{0} / 2}} e_{b}^{* *}\left(S^{*}\right)$. This proves the claim.

It follows that for every $b \in \mathcal{B}=\bigcup_{m=1}^{\infty} \mathcal{B}_{m}, e_{b}^{* *} \in Y^{* *}$. Hence $\ell^{2}(\mathcal{B})$ is contained in $Y^{* *}$, and since $X^{* *}=X \oplus \ell^{2}(\mathcal{B})$, we obtain that $Y^{* *}=Y \oplus \ell^{2}(\mathcal{B})$. Therefore $Y^{* *}$ is not WCG.

To see that $X / Y$ is reflexive we simply observe that

$$
(X / Y)^{* *}=X^{* *} / Y^{* *}=\left(X \oplus \ell^{2}(\mathcal{B})\right) /\left(Y \oplus \ell^{2}(\mathcal{B})\right)=X / Y,
$$

and this completes the proof of the theorem.

Remark 4.4. The unconditional counterpart of the space $X$ is also defined. Namely for a tree $T$ we consider the following norm. For $x \in c_{00}(T)$ we define

$$
\|x\|_{1,2}=\sup \left(\sum_{i=1}^{n}\left(\sum_{t \in S_{i}}|x(t)|\right)^{2}\right)^{1 / 2}
$$

where the supremum is taken over all finite families $\left\{S_{i}\right\}_{i=1}^{n}$ of disjoint segments of $T$. We set $X_{1,2}(T)$ to be the completion of $\left(c_{00}(T),\|\cdot\|_{1,2}\right)$ and clearly $X_{1,2}(T)$ has an unconditional basis. For the sequence $\left(T_{m} \times\{m\}\right)_{m}$ used in the previous example we define $\tilde{X}=\left(\sum_{m=1}^{\infty} \oplus \tilde{X}_{m}\right)_{2}$. Clearly $\tilde{X}$ is WCG and it has an unconditional basis. The subspace $\tilde{Y}$ is defined as in the case of $X$ and also it is not WCG. For every $m \in \mathbb{N}$ and $b \in \mathcal{B}_{m}, b=\left(t_{k}\right)_{k}$, the sequence $\left(e_{t_{k}}\right)_{k}$ is isometric to the usual basis of $\ell^{1}$. It is known that $\left(\ell^{1}\right)^{* *}=\ell^{1} \oplus W$. For $b \in \mathcal{B}=\bigcup_{m} \mathcal{B}_{m}$, we denote by $W_{b}$ the corresponding part of the second dual of $\overline{\operatorname{span}}\left\{\left(e_{t_{k}}\right)_{k}\right\}$. Then

$$
(\tilde{X})^{* *}=(\tilde{X}) \oplus\left(\sum_{b \in \mathcal{B}} \oplus W_{b}\right)_{2} .
$$

Using similar arguments as in the proof of Claim III of Theorem 4.3 we conclude that $\tilde{X} / \tilde{Y}$ is reflexive. 


\section{REFERENCES}

[A] S.A. Argyros, Weakly Lindelöf Determined Banach spaces not containing $\ell^{1}(\mathbb{N}),(1993)$, preprint.

[ACGJM] S.A. Argyros, J.F. Castillo, A.S. Granero, M. Jimenez and J.P. Moreno, Complementation and embeddings of $c_{0}(I)$ in Banach spaces, Proc. London Math. Soc. (3) 85, (2002), 742-768. MR 2003k:46022

[AL] D. Amir and J. Lindenstrauss, The structure of weakly compact sets in Banach spaces, Annals of Math., 88, (1968), 35-46. MR 37:4562

[AM] S.A. Argyros and S. Mercourakis, On weakly Lindelöf Banach spaces, Rocky Mountain J. Math. 23, (1993), 395-446. MR 94i:46016

[F] M.J. Fabián, Gâteaux Differentiability of Convex Functions and Topology, John Wiley \& Sons, Inc. (1997). MR 98h:46009

[F1] M.J. Fabián, Each weakly countably determined Asplund space admits a Fréchet differentiable norm, Bull. Australian Math. Soc., 36, (1987), 367-374. MR 89c:46018

[H] R.G. Haydon, Some more characterizations of Banach spaces containing $\ell^{1}$, Math. Proc. Cambridge Phil. Soc., 80, (1976), 269-276. MR 54:11031

[HHZ] P. Habala, P. Hajek, and V. Zizler, Introduction to Banach Spaces I, II Matfyzpress, Prague (1996).

[HO] J. Hagler and E. Odell, A Banach space not containing $\ell^{1}$ whose dual ball is not weak* sequentially compact, Illinois J. Math. 22, (1978), 290-294. MR 58:2174

[J] R.C. James, A separable somewhat reflexive Banach space with nonseparable dual, Bull. Amer. Math. Soc. 80, (1974), 738-743. MR 54:5811

[JL] W.B. Johnson and J. Lindenstrauss, Some remarks on weakly compactly generated Banach spaces, Israel J. Math. 17, (1974), 219-230. MR 54:5808

[JZ] K. John and V. Zizler, Smoothness and its equivalents in weakly compactly generated Banach spaces, J. Funct. Anal. 15, (1974), 161-166. MR 54:5807

[LS] J. Lindenstrauss and C. Stegall, Examples of separable spaces which do not contain $\ell^{1}$ and whose duals are nonseparable, Studia Math. 54, (1975), 81-105. MR 52:11543

[MS] S. Mercourakis and E. Stamati, A new class of weakly $\mathcal{K}$-analytic Banach spaces, Mathematika (to appear).

[R] H.P. Rosenthal, The heredity problem for weakly compactly generated Banach spaces, Compos. Math. 28, (1974), 83-111. MR 54:5810

[T] M. Talagrand, Espaces de Banach faiblement $\mathcal{K}$-analytiques, Ann. of Math. 110, (1979), 407-438. MR 81a:46021

[Z] V. Zizler, Nonseparable Banach Spaces, Handbook of the Geometry of Banach spaces W.B. Johnson, J. Lindenstrauss (eds.), North-Holland, Vol. 2, ch. 41, (2003), 17431816 .

Department of Mathematics, National Technical University of Athens, Athens 15780, GREECE

E-mail address: sargyros@math.ntua.gr

Department of Mathematics, University of Athens, Athens 15784, Greece

E-mail address: smercour@math.uoa.gr 